\title{
Impact of the gravity field on stability of premixed flames propagating between two closely spaced parallel plates
}

\author{
Daniel Fernández-Galisteo, Vadim N. Kurdyumov* \\ Department of Energy, CIEMAT, Avda. Complutense 40, 28040 Madrid, Spain
}

\begin{abstract}
The stability of a planar flame front propagating between two parallel adiabatic plates inclined at an arbitrary angle is investigated in the frame of narrow-channel approximation. It is demonstrated that buoyancy forces can suppress the hydrodynamic (Darrieus-Landau) and cellular (diffusive-thermal) instabilities for sufficiently large value of the gravity parameter for the case of downward-propagating flames. The stability analysis reveals that in the case of oscillatory diffusive-thermal instability, the flame front can not be stabilized in the similar way. Finally, the stability results are compared satisfactorily with unsteady numerical simulations.
\end{abstract}

\section{Introduction}

Investigations of flame-fluid interactions and buoyancy effects are of practical interest in the design of propulsion devices and is of great importance in understanding the deflagrationto-detonation transition. It must be admitted that direct observations of these phenomena and precise quantitative measurements run into difficulties due to evidence of experimental complexity. These difficulties induce to carry out experiments using special geometric configurations such as narrow channels, for example, where possibility of direct flame observations are of great benefit. Nevertheless some aspects of the flame dynamics, for example the

\footnotetext{
*Corresponding author:

Email address: vadim.k@ciemat.es (Vadim N. Kurdyumov)

Published Journal Article available online: https://doi.org/10.1016/j.proci.2018.06.073
} 
influence of the gravity field (buoyancy forces), are partially suppressed in narrow-channel environment precisely due to its narrowness.

Alternative possibility is observation of flame dynamics between two closely placed parallel plates (made from a transparent material) often referred as a Hele-Shaw cell. Setups of this kind were used in various experiments [1-3] where visualizations of the dynamical flame structures achieved for different mixtures were very promising.

The first theoretical results on the stability of premixed flames propagating between two parallel plates in the presence of gravity can be traced back to the Joulin and Sivashinsky's work [4], where the classical hydrodynamic Darrieus-Landau model treating the flame as a hydrodynamic discontinuity was expanded by including wall effects adding to the inviscid momentum equation the Darcy-like friction force. As a result, instabilities associated with the transport processes in the finite flame structure were neglected. In $[5,6]$ the 2D compressible reactive Navier-Stokes equations were investigated numerically using the phenomenological Poiseuille-like flow assumption. These studies focused primarily on computing the linear growth rates of the instabilities and discussing the flame shapes, although gravity effects were not considered.

In the recent publication [7] a simplified model to describe the flame propagation between two parallel plates was suggested. The model is based on the asymptotic limit of narrow channels explored previously in [8]. This limit assumes $h / \delta_{T} \ll 1$, where $h$ and $\delta_{T}$ are the channel and thermal flame thicknesses, respectively. It allows to simplify the mathematical problem and to gain fundamental understanding that would have otherwise require lengthy numerical simulations. Application of the above assumption for the flame propagation between two parallel plates separated by a narrow gap results in a drastic reduction of complexity of formulation from 3D to 2D. It should be emphasized that the asymptotic considerations can provide approximations and models that could very well extend beyond their strict limit of validity. This statement was confirmed in [9], demonstrating that the narrow-channel approximation works well up to $h / \delta_{T} \approx 5$.

The objective of the present study is to examine the impact of buoyancy forces on the stability of flame propagation between two closely spaced adiabatic plates in the framework 


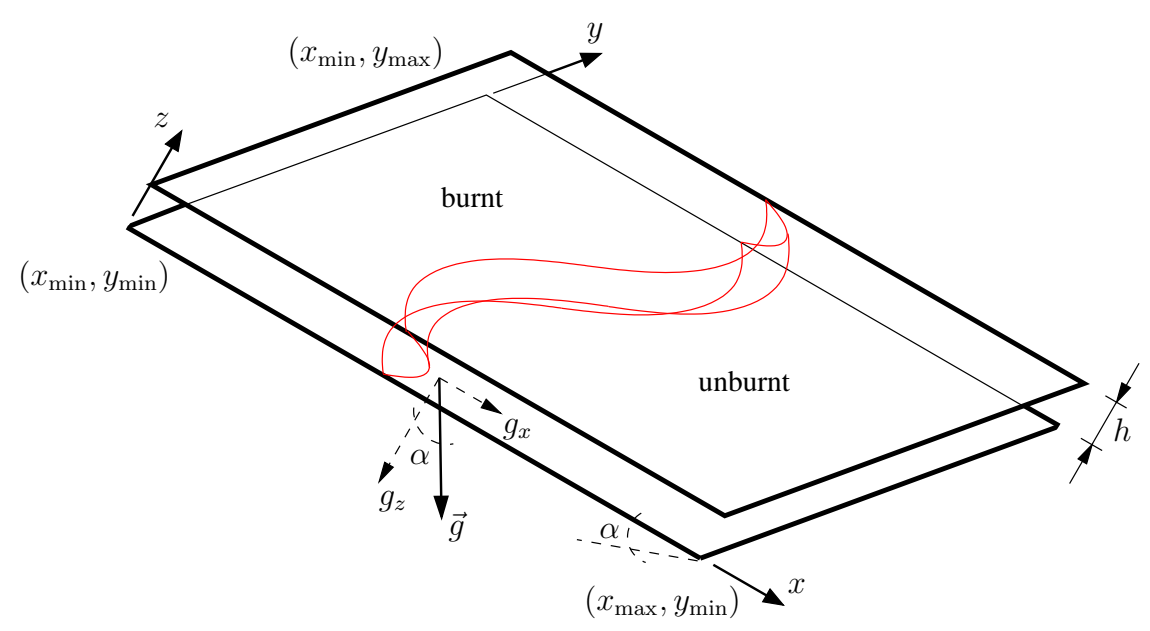

Figure 1: Sketch of the problem and coordinate system.

of the narrow-channel approximation including differential-diffusion effects.

\section{General formulation}

A combustible mixture at rest of uniform density and temperature is contained between two parallel adiabatic plates. The sketch of the problem and the coordinate system are shown in Fig. 1. The gap between the plates, $h$, is considered to be significantly smaller than the plate dimensions in the $x$ and $y$ directions. When the mixture is ignited near the left edge of the plates, at $x=x_{\min }$, a flame propagates towards $x_{\max }$. If one of two diaphragms containing the mixture at $x_{\min }$ or $x_{\max }$ is removed instantaneously upon ignition, the gas here becomes exposed to atmospheric pressure and is allowed to leave through this gap freely. Concerning the lateral boundary conditions situated at $y_{\min }$ and $y_{\max }$, we assume that all variables are periodic here.

The plates can be inclined at arbitrary angle $\alpha$ with the horizontal plane. The components of the gravity acceleration are $g_{x}=|\vec{g}| \sin \alpha$ and $g_{z}=-|\vec{g}| \cos \alpha$ in $x$ and $z$ directions, respectively. A positive value of $\alpha$ corresponds to flames propagating downwards (the burnt hot gas is above the fresh cold one), as shown in Fig. 1, while for negative $\alpha$ the flame propagates upwards. The case $\alpha=0$ corresponds to a flame propagating horizontally. 
The chemical reaction is modeled by a global irreversible step of the form Fuel + Oxidizer $\rightarrow$ Products. For a lean mixture the changes in the oxidizer mass fraction during combustion remain small. The mass of fuel consumed per unit volume and unit time is given by an Arrhenius law of the form, $\tilde{\omega}=\mathcal{B} \tilde{\rho}^{2} Y e^{-E / \mathcal{R} \tilde{T}}$, where $E$ is the overall activation energy of the chemical reaction, $\tilde{T}$ is the temperature, $Y$ is the fuel mass fraction, $\mathcal{R}$ is the gas constant, and $\mathcal{B}$ is an appropriately defined pre-exponential factor. Influence of the oxidizer consumption will be reported elsewhere. We assume in the following that all thermodynamics and transport coefficients are constant together with the well-known low Mach number approximation.

Let us use the laminar flame speed $S_{L}$ of a planar adiabatic and isobaric flame in order to define dimensionless variables. The thickness $h$ is used to scale the transverse coordinate $z$ while the thermal thickness $\delta_{T}=\mathcal{D}_{T} / S_{L}$, with $\mathcal{D}_{T}$ the thermal diffusivity, to scale the coordinates $x, y$. We use $S_{L}$ to scale the gas velocity components in $x$ and $y$ directions and $a S_{L}$ for the z-component and the state of unburned gas $\rho_{u}, p_{u}, T_{u}$ and $Y_{F u}$ for the density $\rho$, pressure $p$, temperature $T$ and the fuel mass fraction $Y$. Using a tilde symbol to denote dimensional variable, we define the following non-dimensional variables

$$
\begin{aligned}
x & =\tilde{x} / \delta_{T}, y=\tilde{y} / \delta_{T}, z=\tilde{z} / h, t=\tilde{t} S_{L} / \delta_{T}, \\
\rho & =\tilde{\rho} / \rho_{u}, u=\tilde{u} / S_{L}, v=\tilde{v} / S_{L}, w=\tilde{w} /\left(a S_{L}\right), \\
p & =a^{2}\left(\tilde{p}-p_{a t m}\right) /\left(12 \operatorname{Pr} \rho_{u} S_{L}^{2}\right), \\
\theta & =\left(\tilde{T}-T_{u}\right) /\left(T_{a}-T_{u}\right), Y=Y_{F} / Y_{F u} .
\end{aligned}
$$

Here $T_{a}=T_{u}+Q Y_{F u} / c_{p}$ is the adiabatic temperature, with $Q$ the heat of combustion per unit mass of fuel and $c_{p}$ is the mixture heat capacity. The parameter $a=h / \delta_{T}$ represents the width of the channel in units of the flame thickness $\delta_{T}$. The factor $12 \operatorname{Pr}$, where $\operatorname{Pr}=\mu / \rho_{u} \mathcal{D}_{T}$ is the Prandtl number based on the viscosity of the mixture $\mu$, is introduced to scale the pressure for the sake of future convenience.

Anticipating the dimensionless equations presented below the following non-dimensional parameters appear in the formulation: the Lewis number, $L e=\mathcal{D}_{T} / \mathcal{D}$, with $\mathcal{D}$ the mass diffusivity of fuel, the heat release parameter, $q=\left(T_{a}-T_{u}\right) / T_{u}=Q Y_{F u} / c_{p} T_{u}$, the dimension- 
less activation energy, $N=E / \mathcal{R} T_{u}$, the dimensionless thickness between plates, $a=h / \delta_{T}$ and the gravity parameter, $G=a^{2}|\vec{g}| \delta_{T} \sin \alpha /\left(12 \operatorname{Pr} S_{L}^{2}\right)$. The standard Zel'dovich number, $\beta=E\left(T_{a}-T_{u}\right) / \mathcal{R} T_{a}^{2}$, commonly used in the asymptotic studies is given by $\beta=N q /(1+q)^{2}$.

The aim of the present study is to investigate the flame propagation in the asymptotic limit $a=h / \delta_{T} \ll 1$. For this purpose all independent variables are expanded in the form $f=f_{0}+a^{2} f_{1}+\ldots$ for a generic variable $f$. This procedure, described in detail in $[7,8]$, represents a regular expansion and is not reproduced here. We give below only the details related with the momentum equations in $x, y$ and $z$ directions which, to leading order, yield

$$
-p_{x}+\frac{1}{12} u_{z z}+\rho G=0,-p_{y}+\frac{1}{12} v_{z z}=0, p_{z}=0
$$

where subscripts denote partial differentiation. The last equation requires $p=p(x, y, t)$ and, then,

$$
u=6 U(x, y, t) z(1-z), v=6 V(x, y, t) z(1-z)
$$

where

$$
U=-p_{x}+\rho G, \quad V=-p_{y}
$$

are the mean velocities in $x$ and $y$ directions, i.e. $(U, V)=\int_{0}^{1}(u, v) \mathrm{d} z$. Remember that the gravity parameter $G$ contains $\sin \alpha$ as a factor. Then, $G$ is positive when the flame propagates downwards $(\alpha>0)$, negative when the flame propagates upwards $(\alpha<0)$, and it is zero for horizontal plates $(\alpha=0)$.

Physically, the above procedure consists in an averaging formulation across the narrow gap so Eqs. (4) resemble the Darcy's law. This limit, strictly speaking, comprises an intermediate asymptotic when, despite $a \rightarrow 0$, the gravity parameter $G$ containing $a^{2}$ remains finite.

The rest of the conservation equations, namely, the mass, the energy and the reactant ones, are reduced to

$$
\begin{gathered}
\rho_{t}+[\rho U]_{x}+[\rho V]_{y}=0 \\
\rho \theta_{t}+\rho U \theta_{x}+\rho V \theta_{y}=\Delta \theta+\omega \\
\rho Y_{t}+\rho U Y_{x}+\rho V Y_{y}=L e^{-1} \Delta Y-\omega
\end{gathered}
$$


where $\Delta=\partial^{2} / \partial x^{2}+\partial^{2} / \partial y^{2}$. These equations should be solved together with Eqs. (4) and the equation of state

$$
\rho(1+q \theta)=1
$$

Appropriate boundary conditions corresponding to flame propagating in the positive $x$-direction between the adiabatic plates are

$$
\begin{array}{ll}
x \rightarrow-\infty: & \theta-1=Y=0 ; \\
x \rightarrow \infty: & \theta=Y-1=0 .
\end{array}
$$

Depending on the configuration the flame can propagate "from" or "to" a closed end, so the boundary conditions for the velocity require

$$
U=0, \quad \text { at } x \rightarrow-\infty \text { or }+\infty
$$

respectively.

The non-dimensional reaction rate term appearing in Eq. (6)-(7) takes the form

$$
\omega=\frac{\beta^{2}}{2 L e u_{p}^{2}} \frac{(1+q)^{2}}{(1+q \theta)^{2}} Y \exp \left\{\frac{\beta(\theta-1)}{(1+q \theta) /(1+q)}\right\} .
$$

The factor $u_{p}=S_{L} / U_{L}$ appearing in Eq. (11) has been introduced to account for the difference between the asymptotic $(\beta \gg 1)$ value of the laminar isobaric flame speed,

$$
U_{L}=\sqrt{2 \mathcal{B} L e \beta^{-2} \frac{\kappa}{c_{p}}} \frac{\rho_{b}}{\rho_{u}} \exp \left(-E / 2 \mathcal{R} T_{a}\right)
$$

with $\rho_{b}=\rho_{u} T_{u} / T_{a}$ the density of the burned gas and $\kappa$ the thermal conductivity, and the value of $S_{L}$ for a finite $\beta$. The factor $u_{p}$ ensures that for a given $\beta$ the non-dimensional speed of a planar adiabatic flame equals one. Clearly, the factor $u_{p}$ tends to one when $\beta \rightarrow \infty$. The numerical value of $u_{p}$, for a given $\beta$ and $L e$, is determined as the eigenvalue of the following one-dimensional problem

$$
\begin{gathered}
-\theta_{\xi}=\theta_{\xi \xi}+\omega, \quad-Y_{\xi}=L e^{-1} Y_{\xi \xi}-\omega \\
\xi \rightarrow+\infty: \theta=Y-1=0 \\
\xi \rightarrow-\infty: \theta-1=Y=0
\end{gathered}
$$


as discussed in [8], with $\omega$ given by Eq. (11). For $\beta=10$ and $q=5$ (the dimensionless activation energy is $N=72$ ), kept as representative values in the present study, $u_{p}=1.0851$, 1.0547 and 0.8458 for $L e=0.7,1$ and 4, respectively. The influence of $\beta$ and $q$ will be reported elsewhere.

Combining Eqs. (5), (6) and (8) one gets

$$
U_{x}+V_{y}=q[\Delta \theta+\omega]
$$

and after using Eq. (4) the following equation for the pressure field can be derived

$$
\Delta p=G \rho_{x}-q[\Delta \theta+\omega]
$$

The pressure field can be excluded from the formulation as follows. Differentiation of Eq. (13) with respect to $x$ gives

$$
U_{x x}+V_{x y}=q[\Delta \theta+\omega]_{x}
$$

Using Eq. (4), the second term in the left-hand side of (15) becomes

$$
V_{x y}=-p_{x y y}=-(\rho G-U)_{y y}=U_{y y}-G \rho_{y y} .
$$

Finally, Eq. (15) takes the form

$$
U_{x x}+U_{y y}-G \rho_{y y}=q[\Delta \theta+\omega]_{x} .
$$

Similar manipulations with $V$ leads to

$$
V_{x x}+V_{y y}+G \rho_{x y}=q[\Delta \theta+\omega]_{y} .
$$

Equation (17) will be used below in the stability analysis.

\section{Planar traveling-wave solution}

In order to describe the flame propagation, a reference frame attached to the flame is applied in the following manner $[10,11]$. Consider a line parallel to the wall and the $x$ axis, $y=$ const., $z=$ const. Following the temperature distribution along this line, starting 
from unburned side, we choose a point $x=x_{*}$ (the first point if there are more than one) where the temperature is equal to some value $\theta=\theta_{*}$ (the reference temperature below). In the following, the reference frame is attached to this point. The dimensionless velocity $u_{f}=U_{f} / S_{L}$ as a function of time of this point relative to the wall, where $U_{f}$ is the dimension flame velocity, characterizes the real time dependent development of the combustion process. Clearly, the specific form of $u_{f}(t)$ depends on the choice of the reference temperature $\theta_{*}$ and its location. However in the case of steady flame propagation the whole flame surface propagates with a constant velocity equal to $u_{f}$.

In the reference frame moving with the flame front, $x \rightarrow x-u_{f} t$, Eqs. (5)-(7) take the form

$$
\begin{gathered}
\rho_{t}+\left[\rho\left(U-u_{f}\right)\right]_{x}+[\rho V]_{y}=0, \\
\rho \theta_{t}+\rho\left(U-u_{f}\right) \theta_{x}+\rho V \theta_{y}=\Delta \theta+\omega, \\
\rho Y_{t}+\rho\left(U-u_{f}\right) Y_{x}+\rho V Y_{y}=L e^{-1} \Delta Y-\omega,
\end{gathered}
$$

while Eqs. (13)-(14) remain unaltered. The instantaneous values of $u_{f}(t)$ are determined by the additional condition

$$
\theta\left(x_{*}, y_{*}, t\right)=\theta_{*}
$$

where $\theta_{*}$ is the non-dimensional reference temperature and $\left(x_{*}, y_{*}\right)$ is the reference point.

Equations (19)-(21) possess a planar solution in the form of a traveling wave where all variables are functions of $x$ exclusively. Then, the continuity equation (19) implies

$$
\rho\left(U-u_{f}\right)=C
$$

where $C$ is a constant. Consequently, Eqs. (20)-(21) are reduced to

$$
C \theta_{x}=\theta_{x x}+\omega, \quad C Y_{x}=L e^{-1} Y_{x x}-\omega,
$$

where direct comparison with Eqs. (12) gives $C=-1$. The mean flow velocity $U$ is thus given by

$$
U=u_{f}-(1+q \theta) .
$$




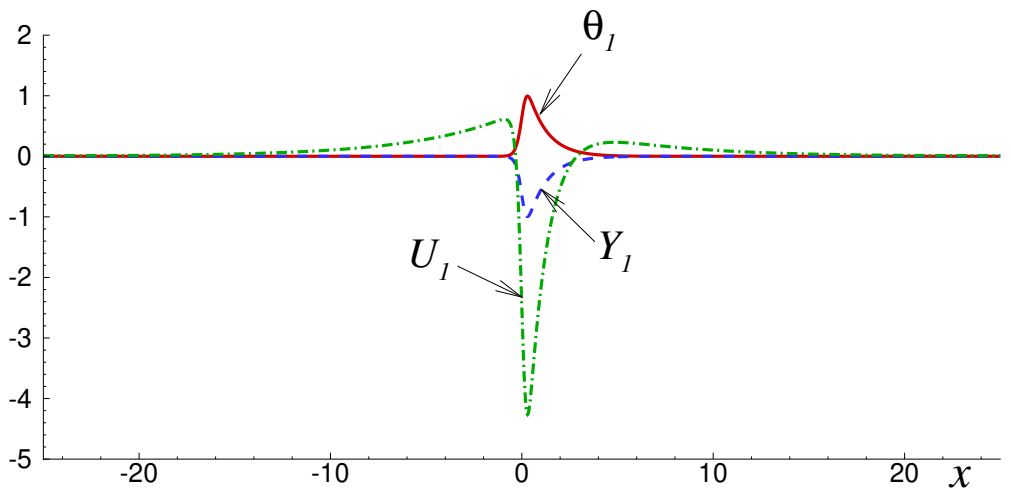

Figure 2: Typical distribution of the eigenfunction plotted for $G=0, k=0.2, L e=1, \beta=10$ and $q=5$.

Equation (25) facilitates to consider two difference cases. When the flame front propagates to a closed end, there is no gas motion far ahead the flame. Then $U=0$ at $x \rightarrow \infty$, while $\theta=0$ here, implying that $u_{f}=1$. When the flame propagates from a closed end, namely $U=0$ at $x \rightarrow-\infty$, condition $\theta=1$ behind the flame implies $u_{f}=1+q$. Notice that in both cases the temperature and mass fraction profiles considered in the moving reference frame remain unaltered and completely identical to a planar flame front. The steady gas velocity profile with respect to the wall becomes $U=-q \theta$ in the first case and $U=q(1-\theta)$ in the second one.

\section{Stability of a planar flame front}

The steady-state solutions corresponding to a planar traveling wave described in the previous section are denoted below by subindex "0". Without loss of generality the steadystate temperature, mass fraction and flow velocity are perturbed in the form

$$
f=f_{0}(x)+\epsilon\left\{f_{1}(x) \exp (i k y)+c f_{0}^{\prime}(x)\right\} \exp (\lambda t)
$$

where $f$ stands for the temperature $\theta$, the mass fraction $Y$ and the velocity components $U$ and $V$. Here $\lambda$ is a complex number, the real part of which represents the growth rate, $k$ is the transverse wave number and $\epsilon$ is a small amplitude. Notice that $V_{0} \equiv 0$ should be used 
in Eq. (26). The flame propagation velocity is also perturbed as follows

$$
u_{f}=u_{f 0}+\epsilon u_{f 1} \exp (\lambda t)
$$

The last term appearing in (26) corresponds to a particular solution of the system (20)(21) and is included in order to take into account the flame propagation velocity perturbation. Details of the procedure was explained in [10]. Substituting Eq. (26) written for the temperature field into Eq. (22) defining the reference frame gives

$$
\theta_{1}\left(x_{*}\right) \exp \left(i k y_{*}\right)+c \theta_{0}^{\prime}\left(x_{*}\right)=0
$$

This equation determines the factor $c$ included in the form of perturbations (26).

The linearized eigenvalue equations obtained when substituting Eq. (26) into Eqs. (20)(21) and (17) are reduced to

$$
\begin{gathered}
\lambda \rho_{0} \theta_{1}-\theta_{1}^{\prime}+\rho_{0}\left\{U_{1}+q \theta_{1}\right\} \theta_{0}^{\prime}= \\
\theta_{1}^{\prime \prime}-k^{2} \theta_{1}+A \theta_{1}+B Y_{1}, \\
\lambda \rho_{0} Y_{1}-Y_{1}^{\prime}+\rho_{0}\left\{U_{1}+q \theta_{1}\right\} Y_{0}^{\prime}= \\
L e^{-1}\left(Y_{1}^{\prime \prime}-k^{2} Y_{1}\right)-A \theta_{1}-B Y_{1}, \\
U_{1}^{\prime \prime}-k^{2} U_{1}=G q k^{2} \rho_{0}^{2} \theta_{1} \\
+q\left\{\theta_{1}^{\prime \prime}-k^{2} \theta_{1}+A \theta_{1}+B Y_{1}\right\}^{\prime}, \\
u_{f 1}=c \lambda,
\end{gathered}
$$

where the primes denote here and below the differentiation with respect to $x$ and

$$
\begin{gathered}
B=\frac{\beta^{2}}{2 L e u_{p}^{2}} \frac{(1+q)^{2}}{\left(1+q \theta_{0}\right)^{2}} \exp \left\{\frac{\beta\left(\theta_{0}-1\right)}{\left(1+q \theta_{0}\right) /(1+q)}\right\}, \\
A=\left\{\frac{\beta(1+q)^{2}}{\left(1+q \theta_{0}\right)^{2}}-\frac{2 q}{1+q \theta_{0}}\right\} B Y_{0},
\end{gathered}
$$

are both functions of $x$. Notice, that the perturbation of $V$ does not enter in Eqs. (29)-(31).

The corresponding boundary conditions read

$$
x \rightarrow \pm \infty: \quad \theta_{1}=Y_{1}=U_{1}=0 .
$$




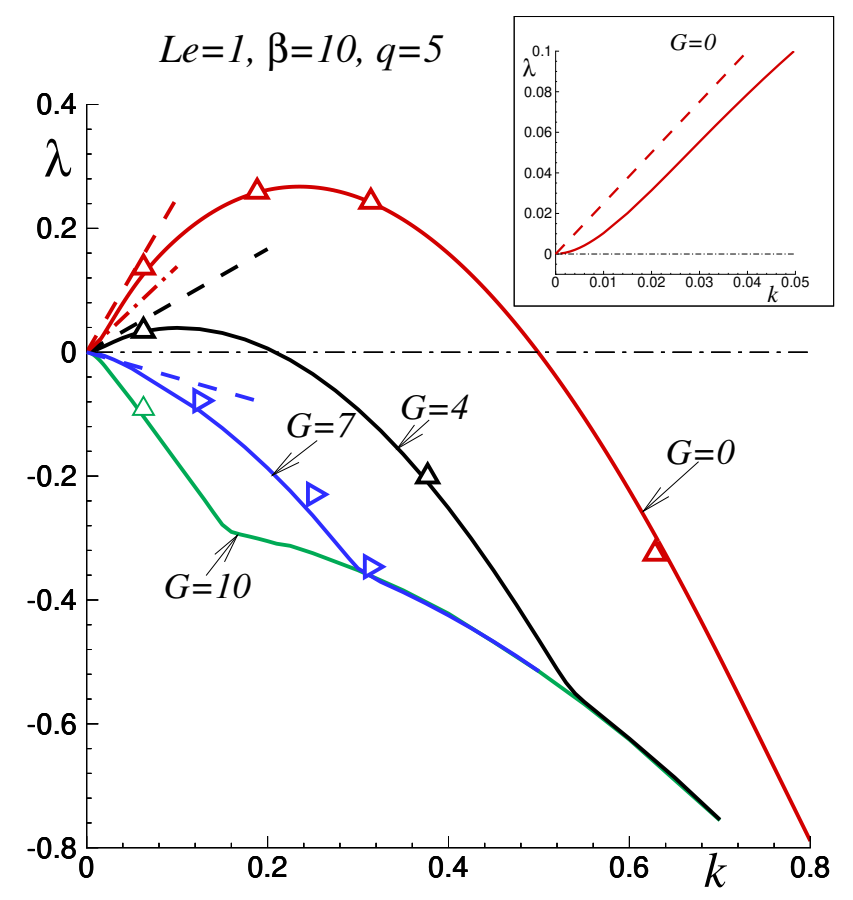

Figure 3: The (real) main eigenvalue $\lambda$ as a function of the wavenumber $k$ calculated for different gravity parameter $G$ and for $L e=1, \beta=10$ and $q=5$; open triangles show the eigenvalues computed form the time-dependent simulations; the dashed lines show the growth rate given by Eq. (34) bases on [4] for $G=0$, 4 and 7 ; the dot-dashed line shows the growth rate for a freely propagating flame, with $G=0$, as given in [14].

It should be emphasized that the eigenvalue $\lambda$ of the stability problem is determined by solving the homogeneous equations (29)-(31), while factor $c$ and the perturbation amplitude of the frame velocity $u_{f 1}$ should be calculated afterwards from Eqs. (28) and (32). Because these profiles are invariant for flame propagation "from" or "to" the close end, see Eqs. (20)(21), the stability properties of a planar flame front in these cases result independent on the case, despite on the differences in the planar flame speed relative to the wall, $u_{f 0}$, equal to 1 or $1+q$ for the "from" or the "to" cases, respectively.

The eigenvalue problem given by Eqs. (29)-(31), together with Eq.(33), was solved using the method described in [10] which allows to calculate the eigenvalue with a largest real part, or the main eigenvalue. If the real part of this eigenvalue is positive, $\lambda_{R}>0$, inside 


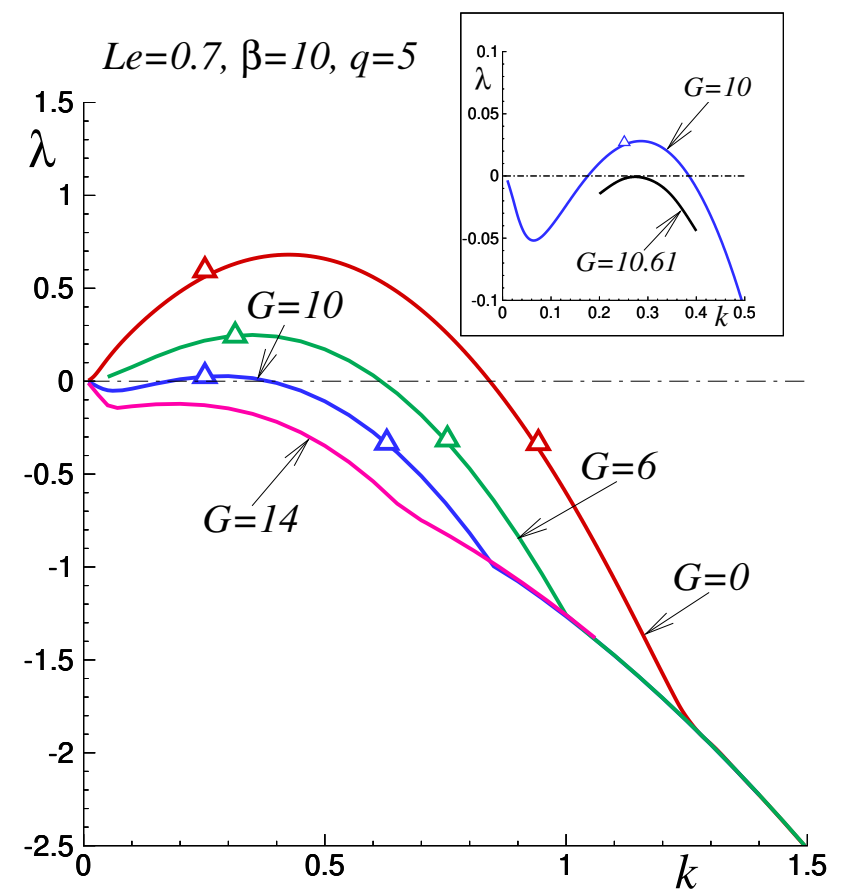

Figure 4: The (real) main eigenvalue $\lambda$ as a function of the wavenumber $k$ calculated for various $G$, all curves for $L e=0.7, \beta=10$ and $q=5$; open triangles show the eigenvalues computed from the time-dependent simulations.

some interval of wavenumber $k$, then the planar flame is unstable, and conversely, if it is non-positive, $\lambda_{R} \leq 0$ for any $k$, the steady state is linearly stable.

Consider first the case $L e=1$ for which the diffusive-thermal mechanism of instability is absent. Numerical calculations show that for $L e=1$ the main eigenvalue and the corresponding eigenfunction are real for all wavenumbers $k$. Figure 2 presents the typical eigenfunction plotted for $G=0, k=0.2, L e=1, \beta=10$ and $q=5$. Notice, that from Eqs. (29)-(30) it follows for $L e=1$ that $Y_{1}=-\theta_{1}$ (together with $Y_{0}^{\prime}=-\theta_{0}^{\prime}$ ).

Figure 3 shows with solid lines the dependence of the growth rate $\lambda$ on the wavenumber $k$ for several values of $G$ calculated for $L e=1, \beta=10$ and $q=5$. One can see that for $G=0$ (horizontal plates) there is a segment of $k$ where the growth rate is positive manifesting the presence of Darrieus-Landau instability. This interval becomes smaller with increasing positive values of $G$ corresponding to the downward flame propagation. The critical value of 
$G$ above which buoyancy forces stabilize the flame is $G_{c} \approx 5.84$. It means that when the hot gas is situated above the flame front buoyancy forces are able to suppress Darrieus-Landau instability.

The analytic analysis carried out in [4] provided the following expression for the growth rate re-written in terms of our variables

$$
\lambda=\frac{q(1+q-G)}{2(1+q)} k .
$$

It can be obtained from a more general dispersion relation (quadratic on $\lambda$ ) to leading order. This dependence is plotted in Fig. 3 with dashed lines for various $G$. One can see a reasonable qualitative agreement manifested for small $k$. Notice that the flame was considered as a hydrodynamic discontinuity in [4] and, as a result, Eq. (34) is independent on the inner flame structure, e.g. the Levis number. Nonetheless the critical value for the flame stabilization reasoning from Eq. (34), $G=6$ (for $q=5$ ), shows a good agreement with $G_{c}=5.84$ obtained in the present study for $L e=1$. For comparison, we also plot in Fig. 3 with dot-dashed line the growth rate of the Darrieus-Landau limit for a freely propagating flame [14].

Consider now the flames with Lewis number lower than one, i.e. $L e=0.7$. Figure 4 exemplifies the (real) growth rate $\lambda$ for various $G$. One can see that buoyancy forces also stabilize the flame above $G=G_{c} \approx 10.61$. It is interesting that the dependence of the growth rate becomes non-monotonic near the threshold value illustrated in the inset of the figure.

Figure 5 displays the stability curves obtained for $L e=4$, where the solid-line segments represent purely real eigenvalues while the dashed-line segments indicate an imaginary counterpart, $\lambda_{I} \neq 0$. The figure shows that for the horizontal case, $G=0$, the flame suffers oscillatory instability and the most unstable mode, corresponding to the maximum of $\lambda_{R}$, is planar $(k=0)$. There can be little doubt that this instability has the diffusive-thermal nature. One can see in Fig. 5 that when the flame propagates downwards, $G>0$, the buoyancy forces are unable to stabilize the flame which remains to be oscillatory unstable with respect to the planar mode $k=0$. It is remarkable that for negative $G$ corresponding to 
the upward flame propagation (the hot gases are below the flame front) the most unstable mode becomes to be monotonic for sufficiently negative $G$.

$$
L e=4, \beta=10, q=5
$$

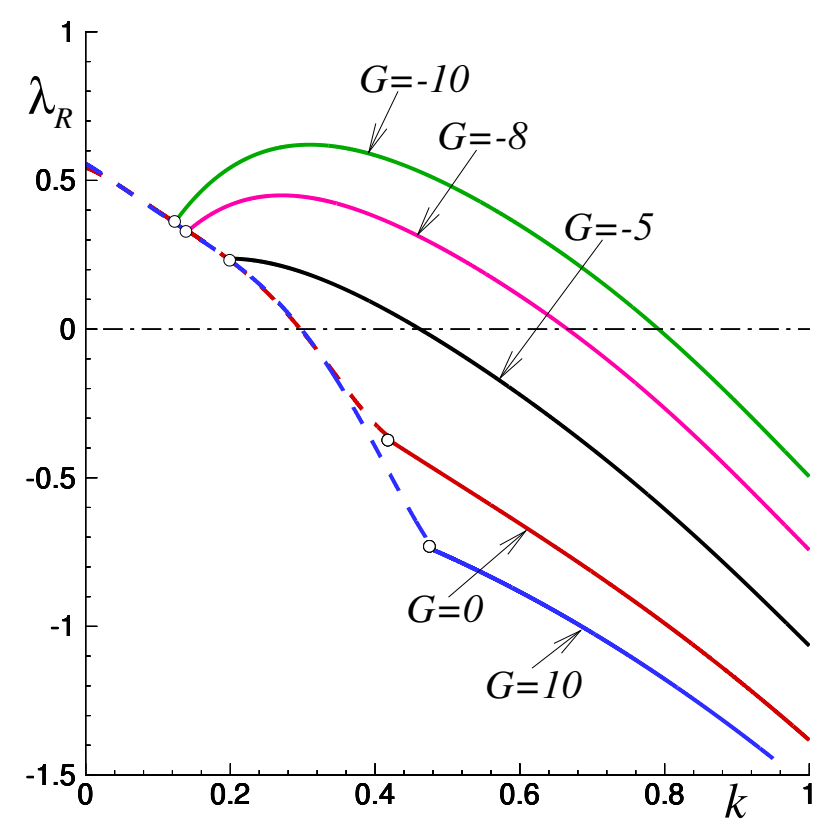

Figure 5: The growth rate $\lambda_{R}$ plotted as a function of $k$ computed for $L e=4, \beta=10$ and $q=5$; for the solid segments $\lambda_{I}=0$ and for dashed segments $\lambda_{I} \neq 0$.

The time dependent problem given by Eqs. (4), (14) and (20)-(21), together with (8), was solved numerically in order to verify the flame stability properties. The initial conditions for simulations were used in the form of a planar flame front with the temperature field perturbed by adding $\varepsilon \exp \left[-\left(x-x_{w}\right)^{2}\right] \sin (k y)$, where the amplitude of perturbations, $\varepsilon$, was of order $10^{-2}, k$ is a fixed wavenumber and $x_{w}$ is a position of the reaction rate maximum. Shown as an example in Fig. 6 are the isolines $\theta=0.9$ plotted at different instants.

The growth rate $\lambda$ calculated from the initial stage of the time evolution was evaluated in the spirit of $[12,13]$. We show in Figs. 3 and 4 with open triangles the calculated values of $\lambda$. One can see a good fit of these results to the linear stability analysis. 


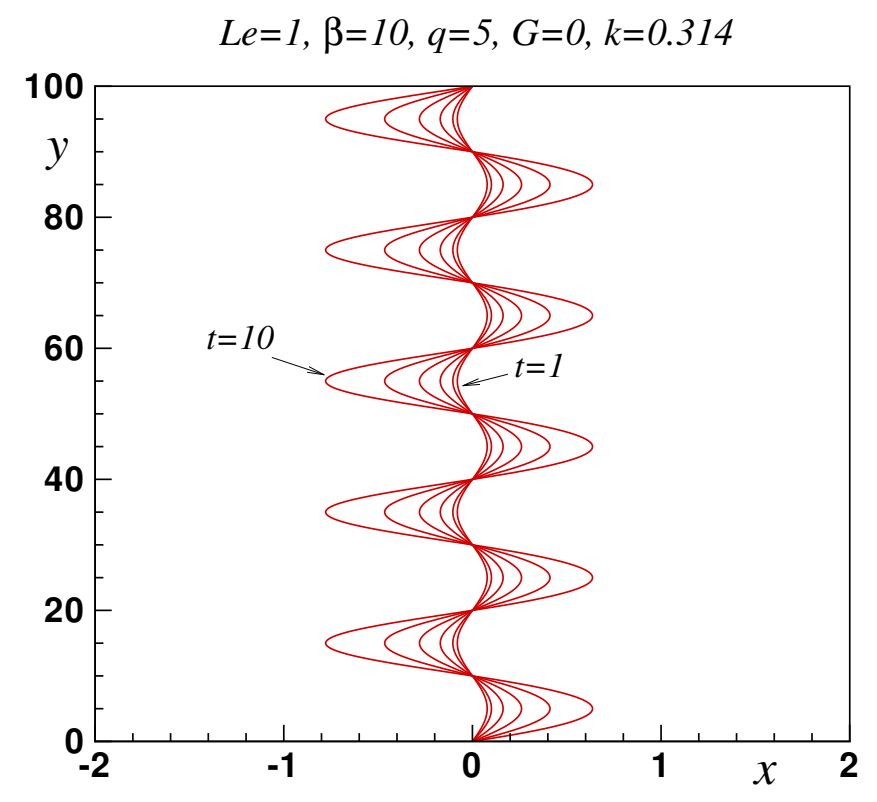

Figure 6: Isoline $\theta=0.9$ plotted at different instants.

\section{Conclusions}

The systematic linear stability analysis of the planar flame front propagating between two parallel adiabatic plates separated by a narrow gap was carried out in the limit of the narrow-channel approximation. In particular, the impact of buoyancy forces was investigated for different values of the Lewis number. The analysis was compared with the initial growth of a periodically perturbed planar flame in time-dependent simulations showing reasonable agreement thus validating the results of the linear stability analysis.

It was found that for downward propagation, when the hot gases (with lower density) are situated above the flame front, buoyancy forces stabilize the hydrodynamic instability for sufficiently large value of the gravity parameter $G=h^{2}|\vec{g}| \sin \alpha /\left(12 \operatorname{Pr} S_{L}^{2} \delta_{T}\right)$. This parameter can be varied, for example, through the angle $\alpha$ of the plate's inclination. The effect of flame stabilization takes place for mixtures with $L e \leq 1$. For the case with $L e=1$ buoyancy forces suppress the hydrodynamic Darrieus-Landau instability only. For mixtures with Le < 1 the hydrodynamic instability is enhanced by the diffusive-thermal (cellular) instability and one needed a higher value of $G$ to get the flame stabilized. Interesting results were 
found for $L e<1$ where stabilization of the smaller wavenumbers together with unstable intermediate wavenumbers occurred. In particular, this effect was observed in experiments of lean hydrogen mixtures propagating downwardly [2], where the large wavelength of wrinkles were stabilized due to buoyancy but the small cell patterns still persisted on the structure.

The stability analysis of the flame front with $L e=4$, characterized by presence of oscillatory diffusive-thermal instability also for a planar perturbation, showed that buoyancy forces are unable to stabilize the intrinsic (diffusive-thermal) oscillations. The most unstable mode corresponds to a planar perturbation with zero wavenumber.

\section{Acknowledgments}

The present work is supported under the project \#ENE2015-65852-C2-2-R (MINECO/FEDER, $\mathrm{EU})$.

[1] J. Sharif, M. Abid, P. D. Ronney, Premixed-gas flame propagation in Hele-Shaw cells, Tech. rep., Spring Technical Meeting, joint U.S. Sections, Combust. Instit. (1999).

[2] J. Wongwiwat, J. Gross, P. D. Ronney, Flame propagation in narrow channels at varying Lewis number, Tech. rep., 25th International Colloquium on the Dynamics of Explosions and Reactive Systems, Paper No. 258 (2015).

[3] C. Almarcha, J. Quinard, B. Denet, E. Al-Sharraf, J. Laugier, E. Villermaux, Phys. Fluids 27 (2015) 91110-91111.

[4] G. Joulin, G. I. Sivashinsky, Combust. Sci. and Tech. 98 (1994) 11-23.

[5] S.H. Kang, H.G. Im, S.W. Baek, Combust. Theory Modell. 7 (2003) 343-363.

[6] S. H. Kang, S. W. Baek, H. G. Im, Combust. Theory Modell. 10 (4) (2006) 659-681.

[7] D. Fernández-Galisteo, V.N. Kurdyumov, P.D. Ronney, Combust. Flame 190 (2018) 133-145.

[8] V.N. Kurdyumov, M. Matalon, Proc. Combust. Inst. 34 (2013) 865-872.

[9] V.N. Kurdyumov, M. Matalon, Proc. Combust. Inst. 35 (2015) 921-928.

[10] V.N. Kurdyumov, Combust. Flame 158 (2011) 1307-1317.

[11] V.N. Kurdyumov, C. Jiménez, Combust. Flame 167 (2016) 149-163.

[12] B. Denet, P. Haldenwang, Combust. Sci. Technol. 104 (1995) 143167.

[13] S. Kadowaki, Phys, Fluids 12 (2000) 2352-2359.

[14] C.K. Law, C.J. Sung, Prog. Energ. Combust. 26 (2000) 459-505. 